\title{
Posttreatment Downregulation of Type III Interferons in Patients with Acute Brucellosis
}

\author{
Mehran Shokri $\mathbb{D}^{1},{ }^{1}$ Oreinab Ghaffari Khonakdar, ${ }^{2}$ Mousa Mohammadnia-Afrouzi $\left(\mathbb{D},{ }^{3}\right.$ \\ Mahmoud Sadeghi-Haddad-Zavareh (D), ${ }^{1}$ Amirhossein Hasanpour, ${ }^{2}$ Mohammad Barary $\mathbb{D}^{2},{ }^{2}$ \\ and Soheil Ebrahimpour $\mathbb{D}^{1}$ \\ ${ }^{1}$ Infectious Diseases and Tropical Medicine Research Center, Health Research Institute, Babol University of Medical Sciences, \\ Babol, Iran \\ ${ }^{2}$ Student Research Committee, Babol University of Medical Sciences, Babol, Iran \\ ${ }^{3}$ Immunoregulation Research Center, Health Research Institute, Babol University of Medical Sciences, Babol, Iran
}

Correspondence should be addressed to Soheil Ebrahimpour; drsoheil1503@yahoo.com

Received 19 May 2021; Revised 6 June 2021; Accepted 29 June 2021; Published 19 July 2021

Academic Editor: Matilde Otero-Losada

Copyright ( 2021 Mehran Shokri et al. This is an open access article distributed under the Creative Commons Attribution License, which permits unrestricted use, distribution, and reproduction in any medium, provided the original work is properly cited.

\begin{abstract}
There is a limited number of clinical studies on interferon (IFN) levels in human brucellosis. The novel group of interferons, type III interferons, which consists of four IFN- $\lambda$ (lambda) molecules called IFN- $\lambda 1$ or interleukin-29 (IL-29), IFN- $\lambda 2$ or IL-28A, IFN- $\lambda 3$ or IL-28B, and IFN- $\lambda 4$, is not fully known. This study is one of the first studies of IL-28A and IL-29 levels in brucellosis cases at the end of their treatment course. A total of 33 acute brucellosis patients were included in this study. We considered changes in the levels of IL-28A and IL-29 in cases with acute brucellosis before and after treatment with standard therapy that referred to the Ayatollah Rohani Hospital in Babol, northern Iran. Of 33 included patients, 22 (66.6\%) were males, and 11 (33.4\%) were females. The range of patients' age was $49.21 \pm 17.70$ years. Serum IL-29 and IL-28A (acute form: $56.4 \pm 30.32 \mathrm{pg} / \mathrm{mL}$ and $48.73 \pm 27.72$ $\mathrm{pg} / \mathrm{mL}$, respectively, and posttreatment: $40.15 \pm 20.30 \mathrm{pg} / \mathrm{mL}$ and $38.79 \pm 22.66 \mathrm{pg} / \mathrm{mL}$, respectively) levels were elevated significantly in acute brucellosis than after treatment $(p<0.05)$. These findings indicate that considering biomarker levels in brucellosis patients may indicate the chronicity of infection. In conclusion, we suggest that IL-29 and IL-28A levels may be valuable biomarkers for follow-up patients with brucellosis.
\end{abstract}

\section{Introduction}

Brucellosis is a zoonotic bacterial disease caused by one of the various species of the Brucella spp. [1-3]. Although approximately half-million new brucellosis cases are reported worldwide, the actual incidence rate has been much more significant $[4,5]$. Even though the gold standard for diagnosing this disease is leukocyte culture, this test has a high falsenegative rate. Moreover, its cost and a 10-day delay before confirmation restrict its use as a standard diagnostic test in acute brucellosis [6]. As a result, clinicians chiefly rely on other laboratory tests to evaluate patients with brucellosis, such as agglutination test, white blood cell (WBC) counts, platelet (PLT) counts, liver function tests, erythrocyte sedimentation rate (ESR), and C-reactive protein (CRP). Never- theless, the diagnosis of brucellosis remains a challenge in most cases [6].

Although rare, brucellosis infection has a chronic disease course that may continue to trouble patients for years. No approved human antibrucellosis vaccine is currently available [7]. So far, brucellosis studies have been focused primarily on epidemiological investigations, and the immune response against these bacteria was somehow neglected [8]. After the entry of this pathogen, several immunogenic changes are prominent in the host body. For example, interferon-gamma (IFN- $\gamma$ ) and tumor necrosis factor-alpha (TNF- $\alpha$ ) levels are elevated as naive T cells differentiate to $\mathrm{CD}^{+}$helper $\mathrm{T}$ cells type $1\left(\mathrm{Th}_{1}\right)$. On the other hand, interleukin-4 (IL-4) levels are increased as naïve $\mathrm{T}$ cells give rise to $\mathrm{CD} 4^{+}$helper $\mathrm{T}$ cells type $2\left(\mathrm{Th}_{2}\right)[6]$. 
Moreover, transforming growth factor-beta (TGF- $\beta$ ) levels are also raised due to the surge in the regulatory $\mathrm{T}$ cell (Treg) population [6]. Several studies have shown that interferon-gamma serum concentration may be recognized as an essential factor in chronic brucellosis [8]. It is vital to note that the association between other types of cytokines, such as type III interferons or interferon-lambda (IFN- $\lambda$ ), and the clinical course of brucellosis, including response to treatment, is not yet fully understood. Nevertheless, some studies recognized that bacterial pathogens might activate IFN- $\lambda 1$ or interleukin-29 (IL-29), IFN- $\lambda 2$ or IL-28A, IFN- $\lambda 3$, or IL-28B $[9,10]$.

Therefore, this study was conducted to evaluate the levels of IL-28A and IL-29 in patients with brucellosis, both pretreatment and posttreatment.

\section{Materials and Methods}

2.1. Patients. In this case-control study at the Ayatollah Rohani Hospital in Babol, northern Iran, 33 pretreatment and posttreatment acute brucellosis patients were included. Inclusion criteria were defined as receiving a clinical diagnosis of acute brucellosis (clinical presentation time: acute form ( $\leq 2$ months) based on the symptoms, compatible clinical findings, standard tube agglutination (STA) test titer $\geq 1: 160$, and the presence of 2-mercaptoethanol (2ME) agglutination $\geq 1: 80$. The control group consisted of the same 33 patients who had undergone a complete course of treatment with gentamicin $5 \mathrm{mg} / \mathrm{kg} /$ day IM for seven days plus doxycycline $100 \mathrm{mg}$ tablet BID for 45 days [11]. Then, the patients were included in the control group and considered treated when no clinical manifestations related to brucellosis and compatible paraclinical findings, i.e., the STA test titer $<1: 160$ and $2 \mathrm{ME}$ agglutination $<1: 80$, were present [12]. The exclusion criteria were pregnancy, age $<18$ years, and other chronic infectious or immunodeficiency diseases. Informed consent was obtained from all study participants.

2.2. Determination of Cytokine Levels. Blood samples were collected in an ethylenediamine tetraacetic acid- (EDTA-) containing tube. One blood sample $(5 \mathrm{~mL})$ was obtained from all patients before and after treatment. The blood samples were centrifuged at $400 \mathrm{~g}$ for 35 minutes, and collected sera were stored at $-80^{\circ} \mathrm{C}$ for further analysis. IL-28A and IL-29 levels in all serum samples were measured using a commercial enzyme-linked immunosorbent assay (ELISA) kit (eBioscience Inc., San Diego, CA, USA) according to the manufacturer's guidelines.

2.3. Statistical Analysis. All data are expressed as the mean \pm standard deviation (SD). Statistical analysis was performed using the SPSS software version 16.0 (IBM, Chicago, IL, USA). The difference between groups was analyzed using the paired $t$-test, and the relationship between variables was evaluated using Spearman's rank correlation test. A $p$ value of $\leq 0.05$ was defined as statistically significant.

\section{Results}

Of 33 included patients, $22(66.6 \%)$ were males, and 11 (33.4\%) were females. The range of patients' age was $49.21 \pm 17.70$ years.

Serum levels of IL-28A and IL-29 in the study groups (before and after the treatment) are illustrated in Figure 1. Serum IL-28A levels were $48.73 \pm 27.72 \mathrm{pg} / \mathrm{mL}$ and $38.79 \pm$ $22.66 \mathrm{pg} / \mathrm{mL}$ pre- and posttreatment, respectively $(p=0.038)$ (Figure 1). Also, IL-29 serum levels were $56.45 \pm 30.32 \mathrm{pg} / \mathrm{mL}$ and $40.15 \pm 20.30 \mathrm{pg} / \mathrm{mL}$ pre- and posttreatment, respectively $(p=0.026)$ (Figure 1). Also, it is noteworthy that IL-29 levels both before and after treatment were more than IL-28A levels.

\section{Discussion}

In this case-control study, the IL-28A and IL-29 levels of 33 patients with confirmed acute brucellosis were measured both pre- and posttreatment. It was observed that the levels of these biomarkers were significantly decreased after the eradication of the disease.

Several studies revealed the crucial role of type III IFNs for resistance to viral infections, principally by induction of the antiviral state. Furthermore, some studies revealed that type III IFNs play a significant role in inhibiting virus replication by mediating and expressing interferon-regulated genes (IRGs) [13]. Subsequent studies have also shown an inhibiting role of interferon in replicating Zika virus (ZIKV), influenza $A$ and $B$ viruses, coronavirus, and respiratory syncytial virus (RSV) $[14,15]$. Ank et al. concluded that interferonlambda plays a crucial role in the innate immune response through activating the macrophages and dendritic cells against human herpesvirus type 1 (HHV-1) [16]. Another study demonstrated dengue virus (DENV) replication inhibition through interferon-regulating gene expression [17].

The role of IL-29 in bacterial and parasitic infections and its increased expression in these diseases have been proven before. Also, the critical role of interferon-lambda has been highlighted in the acquired immune response in previous studies. This cytokine initiates the received immune response through its effect on antigen-presenting cells. Moreover, type III IFNs suppress the immune response through regulatory $\mathrm{T}$ cells (Tregs), promoting the acquired immune response [18]. As shown in previous studies, the human immune system's defense against brucellosis depends on cellular immunity, which mainly affects antigen-presenting cells (APC), such as macrophages, dendritic cells, and $\mathrm{CD} 4^{+}$and $\mathrm{CD} 8^{+} \mathrm{T}$ cells. Other defense cells, such as natural killer (NK) cells and other T lymphocytes, also play a crucial role in cellular immunity against Brucella [19]. As a result, the $\mathrm{Th}_{1}$-dependent immune response is dominant at the onset of Brucella infection and is critical in eradicating the disease. Therefore, this issue fully justifies the current study results, particularly the higher levels of IL-29 in patients with acute brucellosis before treatment than after receiving the standard treatment [19]. Figure 2 illustrates a summary of the immune reactions of the host's body and the vital role of different cytokines, such as IFN- $\gamma$, TNF- $\alpha$, and IFN- $\lambda$, in the eradication of Brucella spp. 


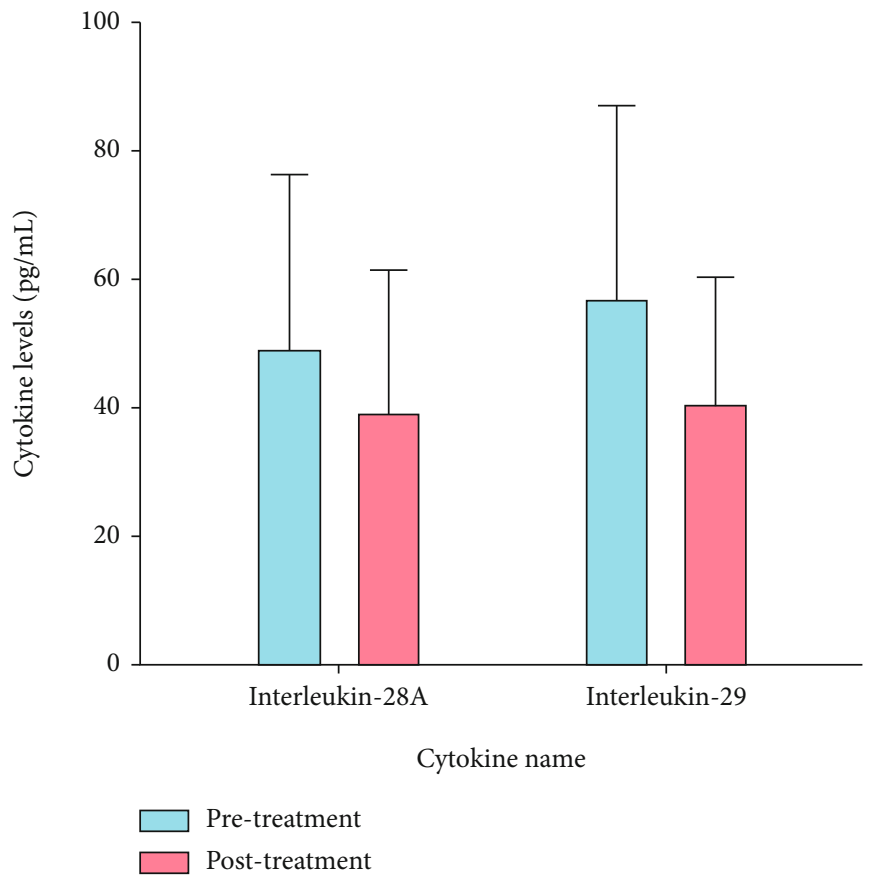

Figure 1: Serum levels of interleukin-28A and interleukin-29 among patients with brucellosis before and after treatment. All data are presented as the mean \pm SD. $*$ indicates $p<0.05$.

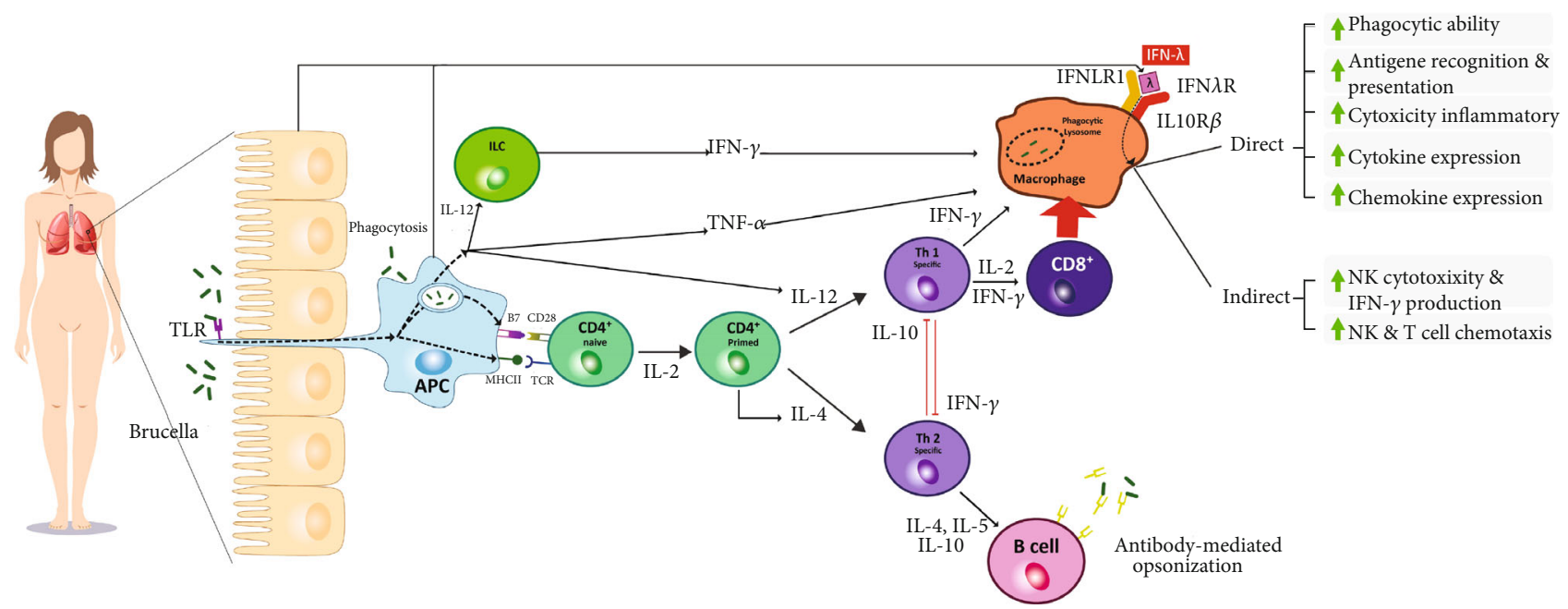

Figure 2: Interactions of Brucella spp. with the immune system. After the activation of antigen-presenting cells (APCs) with Brucella antigen via the Toll-like receptor (TLR) signaling pathway, a cascade of events leads to the priming of CD4 ${ }^{+} \mathrm{T}$ cells to helper $\mathrm{T}$ cells type $1\left(\mathrm{Th} \mathrm{h}_{1}\right)$ and type $2\left(\mathrm{Th}_{2}\right)$. Th 1 cells secrete various cytokines, such as TNF- $\alpha$ and IFN- $\gamma$, which activate and enhance the anti-Brucella mechanisms of macrophages and activate $\mathrm{CD}^{+} \mathrm{T}$ cells, which boost the immune responses of macrophages even further. Moreover, APCs can trigger $\mathrm{Th}_{2}$ activation, which switches on B lymphocytes and the humoral immunity, facilitating the opsonization and faster eradication of the pathogen from the host's body. It is noteworthy that $\mathrm{Th}_{1}$ and $\mathrm{Th}_{2}$ cells can inhibit either pathway via secreting cytokines, such as IFN- $\gamma$ and IL-10, respectively. Furthermore, macrophages can be stimulated by secreting another cytokine called type III interferons or interferon- $\lambda$ by APCs or epithelial cells. These activated macrophages exert their immunomodulatory effects through two different pathways: direct and indirect. In the direct pathway, chemokine and inflammatory cytokine expression, antigen recognition and presentation, and macrophages' cytotoxicity are elevated. Through the indirect pathway, these cells can enhance natural killer (NK) and T cell chemotaxis and NK cell cytotoxicity and elevate the production and release rate of IFN- $\gamma$, which in turn, via activating the Th pathway, helps better and faster eradication of Brucella spp. Abbreviations: TLR: Toll-like receptor; ILC: innate lymphocyte cells; IL-12: interleukin-12; APC: antigen-presenting cells; B7: cluster of differentiation 80/86; MHC II: major histocompatibility complex type 2; CD28: cluster of differentiation 28; TCR: T cell receptor; IFN- $\gamma$ : interferon-gamma; TNF- $\alpha$ : tumor necrosis factor-alpha; IL-4: interleukin-4; $\mathrm{Th}_{1}$ : helper $\mathrm{T}$ cell type $1 ; \mathrm{Th}_{2}$ : helper T cell type 2 ; IL-2: interleukin-2; IL-10: interleukin-10; IL-5: interleukin-5; IFN- $\lambda$ : interferon- $\lambda$; IFNLR1: interferon-lambda receptor 1; IL10R $\beta$ : interleukin-10 receptor beta; IFN $\lambda$ R: interferon-lambda receptor; NK cell: natural killer cell. 
The present study showed a significant decrease in IL-28-A and IL-29 levels after treatment with the standard antibiotic regimen, i.e., gentamicin $5 \mathrm{mg} / \mathrm{kg} /$ day IV for seven days plus doxycycline $100 \mathrm{mg}$ tablet BID for 45 days. Regarding the IL-28A levels, it seems that cross-linking of IL-28A with type I interferons (IFNs) and subsequent innate and acquired immune responses against brucellosis may be the cause. For IL-29, this reduction can be attributed to its vital role in acquired immunity, eradicating brucellosis using antibiotic treatment, and reduced inflammation [19]. IFNs initiate an innate immune response after contact with pathogens. The immune response and immune mediators and the subsequent inflammation are expected to decline following the control and eradication of infection. It is important to note that some standard diagnostic tests, such as STA, Coombs Wright, and $2 \mathrm{ME}$, may remain positive even at high titers for up to two years after treatment [20, 21]. Thus, such tests' application was not justified for follow-up patients. Practically, most patients are generally followed up with their symptoms [22].

The current study results revealed a significant reduction in serum IL-28A and IL-29 levels after treatment, making these biomarkers a valuable indicator for monitoring the patients. While the effects of IL-28A on the acquired immunity are not significant, its functions are chiefly exerted via innate immunity, with no effect on increasing or decreasing immunoglobulins (Ig) [22]. On the other hand, some previous studies have shown a positive correlation of IL-29 with serological tests that indicate its impact on acquired immunity and Ig production. However, it is essential to note that cellular immunity plays a more crucial role in eliminating brucellosis [22].

The primary limitation of this was our small sample size. It is recommended to reperform such studies in a larger sample size. Also, another limitation of the current study was the sole evaluation of interferon-lambda. It is suggested that future researchers assess the levels of other vital cytokines, such as interferon-alpha (IFN- $\alpha$ ) and interferon-gamma (IFN- $\gamma)$, tumor necrosis factor-alpha (TNF- $\alpha$ ), transforming growth factor-beta (TGF- $\beta$ ), interleukin-2 (IL-2), and interleukin-12 (IL-12). Furthermore, performing molecular analysis, i.e., PCR test, would be of interest. Moreover, future studies with a prolonged follow-up assessing these IFNs in different periods could help confirm the role of IFN- $\gamma$ as a reliable biomarker in chronic brucellosis.

\section{Conclusion}

This study's findings confirmed previous studies on bacterial infections and validated the pivotal role of IFN- $\lambda$ during acute brucellosis via strengthening innate and promoting acquired immunities. Therefore, the significant reduction in serum levels of IL-28A and IL-29 in patients with brucellosis after a standard treatment regimen may promise the emergence of valuable biomarkers in patient follow-up.

\section{Data Availability}

The data that support the findings of this study are available from the corresponding author upon reasonable request.

\section{Ethical Approval}

This study protocol was approved by the ethics committee of Babol University of Medical Sciences (IR.MUBABOL.HRI.REC.1397.212).

\section{Conflicts of Interest}

All authors declare no conflict of interest.

\section{Authors' Contributions}

MS designed the research study, collected data, and analyzed data. OGK collected data and helped with manuscript writing. MMA collected data and helped with manuscript writing. MSHZ collected data and helped with manuscript writing. $\mathrm{AH}$ worked on visualization and software. $\mathrm{MB}$ collected data and contributed substantial revisions to the content of all sections of the manuscript. SE designed the research study and contributed substantial revisions to all sections of the manuscript.

\section{Acknowledgments}

The authors thank the Department of Infectious Diseases of Babol University of Medical Sciences. This study was fully supported by the vice-chancellor for research and technology of Babol University of Medical Sciences.

\section{References}

[1] M. Mohammadnia-Afrouzi and S. Ebrahimpour, "Assessment of TGF- $\beta$ and IL10 levels in human brucellosis," Current Issues in Pharmacy and Medical Sciences, vol. 31, no. 1, pp. 22-24, 2018.

[2] Y. Wang, C. Xu, S. Zhang, Z. Wang, Y. Zhu, and J. Yuan, "Temporal trends analysis of human brucellosis incidence in mainland China from 2004 to 2018," Scientific Reports, vol. 8, no. 1, p. 15901, 2018.

[3] G. Pappas, N. Akritidis, M. Bosilkovski, and E. Tsianos, "Medical progress brucellosis," The New England journal of medicine, vol. 352, no. 22, pp. 2325-2336, 2005.

[4] M. R. Hasanjani Roushan and S. Ebrahimpour, "Human brucellosis: an overview," Caspian journal of internal medicine, vol. 6 , no. 1, pp. 46-47, 2015, https://pubmed.ncbi.nlm.nih .gov/26221498.

[5] M. R. Hasanjani Roushan, M. Bayani, S. Soleimaniamiri, M. Mohammadnia-Afrouzi, H. R. Nouri, and S. Ebrahimpour, "Evaluation of CD4+CD25+ FoxP3+ regulatory T cells during treatment of patients with brucellosis," Journal of Biological Regulators and Homeostatic Agents, vol. 30, no. 3, pp. 675682, 2016, http://www.ncbi.nlm.nih.gov/pubmed/27655484.

[6] G. Xu, P. Zhang, R. Dang et al., "Dynamic changes of Th1 cytokines and the clinical significance of the IFN- $\gamma /$ TNF- ratio in acute brucellosis," Mediators of Inflammation, vol. 2019, 10 pages, 2019, https://www.hindawi.com/journals/mi/2019/ $5869257 /$.

[7] S. D. Perkins, S. J. Smither, and H. S. Atkins, "Towards a Brucella vaccine for humans," FEMS Microbiology Reviews, vol. 34, no. 3, pp. 379-394, 2010. 
[8] H. A. N. Li-hong, L. I. U. Ri-hong, and L. I. U. Z.-g. CB, “High level IFN- $\gamma$ may be one of the characteristics of chronic brucellosis," Chinese Journal of Zoonoses, vol. 33, no. 8, pp. 716-719, 2017, http://www.rsghb.cn/EN/abstract/abstract23948.shtml.

[9] T. S. Cohen and D. Parker, "Microbial pathogenesis and type III interferons," Cytokine and Growth Factor Reviews, vol. 29, pp. 45-51, 2016.

[10] H. E. Rich, C. C. McCourt, W. Q. Zheng et al., "Interferon lambda inhibits bacterial uptake during influenza superinfection," in Infection and Immunity, A. J. Bäumler, Ed., vol. 87, no. 5, 2019.

[11] M. R. Hasanjani Roushan, M. Mohraz, M. Hajiahmadi, A. Ramzani, and A. A. Valayati, "Efficacy of gentamicin plus doxycycline versus streptomycin plus doxycycline in the treatment of brucellosis in humans," Clinical Infectious Diseases, vol. 42, no. 8, pp. 1075-1080, 2006.

[12] M. R. Hasanjani Roushan and S. Ebrahimpour, "Human brucellosis: an overview," International Journal of Infectious Diseases, vol. 6, no. 1, pp. 46-47, 2015, http://caspjim.com/ article-1-447-en.html.

[13] K. Pervolaraki, S. Rastgou Talemi, D. Albrecht et al., "Differential induction of interferon stimulated genes between type I and type III interferons is independent of interferon receptor abundance," PLoS Pathogens, vol. 14, no. 11, article e1007420, 2018.

[14] S. V. Kotenko, A. Rivera, D. Parker, and J. E. Durbin, “Type III IFNs: beyond antiviral protection," Seminars in Immunology, vol. 43, article 101303, 2019.

[15] Y. J. Jeon, J. H. Lim, S. An et al., "Type III interferons are critical host factors that determine susceptibility to influenza A viral infection in allergic nasal mucosa," Clinical and Experimental Allergy, vol. 48, no. 3, pp. 253-265, 2018.

[16] N. Ank, H. West, and S. R. Paludan, "IFN- $\lambda$ : novel antiviral cytokines," Journal of Interferon and Cytokine Research, vol. 26, no. 6, pp. 373-379, 2006.

[17] H. K. Palma-Ocampo, J. C. Flores-Alonso, V. Vallejo-Ruiz et al., "Interferon lambda inhibits dengue virus replication in epithelial cells," Virology Journal, vol. 12, no. 1, p. 150, 2015.

[18] I. Misumi and J. K. Whitmire, "IFN- $\lambda$ exerts opposing effects on $\mathrm{T}$ cell responses depending on the chronicity of the virus infection," The Journal of Immunology, vol. 192, no. 8, pp. 3596-3606, 2014.

[19] K. Witte, E. Witte, R. Sabat, and K. Wolk, "IL-28A, IL-28B, and IL-29: promising cytokines with type I interferon-like properties," Cytokine and Growth Factor Reviews, vol. 21, no. 4, pp. 237-251, 2010.

[20] M. R. H. Roushan, M. J. S. Amiri, A. Laly, A. Mostafazadeh, and A. Bijani, "Follow-up standard agglutination and 2mercaptoethanol tests in 175 clinically cured cases of human brucellosis," International Journal of Infectious Diseases, vol. 14, no. 3, pp. e250-e253, 2010.

[21] G. F. Araj, "Update on laboratory diagnosis of human brucellosis," International Journal of Antimicrobial Agents, vol. 36, Supplement 1, pp. S12-S17, 2010.

[22] A. Dolganiuc, K. Kodys, C. Marshall et al., “Type III interferons, IL-28 and IL-29, are increased in chronic HCV infection and induce myeloid dendritic cell-mediated FoxP3+ regulatory T cells," PLoS one, vol. 10, no. 10, article e44915, 2012. 